\title{
Impact of the 7th Edition AJCC Staging Classification on the NCCN Clinical Practice Guidelines in Oncology for Gastric and Esophageal Cancers
}

\author{
Vivian E. Strong, MDª Thomas A. D’Amico, MD ; Lawrence Kleinberg, MD; and Jaffer Ajani, MD
}

\begin{abstract}
The 7th edition of the AJCC Cancer Staging Manual has attempted to harmonize gastric and esophageal cancers, including management of gastroesophageal junction (GEJ)-type tumors. The treatment of complex tumor types is best guided by a staging classification that reliably groups patients according to prognosis and therapy. This article reviews and discusses these changes with the goal of elucidating key features of the staging system and outlining how these changes relate to the NCCN Clinical Practice Guidelines in Oncology with regard to the care and treatment of patients. The 7th edition of the AJCC Cancer Staging Manual has certainly improved harmonization of gastric and distal esophageal/GEJ-type adenocarcinomas, although issues persist, particularly regarding the optimal neoadjuvant treatment for the management of GEJ carcinomas. (JNCCN 2013;11:60-66)
\end{abstract}

The goal of the most recent 7th edition of the AJCC Cancer Staging Manual for gastric and esophageal cancer is to harmonize tumors arising from the distal esophagus to gastric cardia, including management of gastroesophageal junction (GEJ)-type tumors. The treatment of complex tumor types is best guided by a staging classification that reliably groups patients according to prognosis and therapy. The development of

From aMemorial Sloan-Kettering Cancer Center, New York, New York; 'buke University Medical Center, Durham, North Carolina; Johns Hopkins Medical Center, Baltimore, Maryland; and dThe University of Texas MD Anderson Cancer Center, Houston, Texas. Submitted May 5, 2011; accepted for publication

September 8, 2012.

The authors have disclosed that they have no financial interests, arrangements, affiliations, or commercial interests with the manufacturers of any products discussed in this article or their competitors.

Correspondence: Vivian E. Strong, MD, Department of Surgery, Memorial Sloan-Kettering Cancer Center, 1275 York Avenue,

H-1217, New York, NY 10065. E-mail: strongv@mskcc.org effective staging classifications is based on the analysis of large databases, ideally composed of patients that are consistently and accurately staged and treated, to derive accurate staging groups. Many challenges exist to developing a solid staging system for this area, because improved understanding of gastric, esophageal, and GEJ tumors has shown that this is a heterogeneous disease with varying outcomes depending on tumor location and type. Developing a system to adequately address these issues is an evolving target that will continue to require refinement and reconsideration as further data and analysis emerge. Therefore, this article elucidates the key features of this most recent staging system and outlines the changes and how they impact the NCCN Clinical Practice Guidelines for Oncology (NCCN Guidelines) for Gastric and Esophageal Cancer.

\section{Esophageal Cancer}

The staging classification for esophageal cancer has been limited by several factors, including histologic diversity, the inconsistent use of multimodality therapy in operable patients, and the variety of surgical strategies used, which influences the creation of treatment guidelines. The 7th edition of the AJCC Cancer Staging Manual ${ }^{2,3}$ attempts to resolve some of the inconsistencies of previous versions. The modifications of the staging system and the treatment guidelines are described, with specific attention to the most controversial issues.

\section{Tumor ( $\mathrm{T}$ ) Status}

The classification omits the previous designation Tis, which is now defined as high-grade dysplasia (HGD) and includes all noninvasive neoplastic epithelium previously called carcinoma in situ. T4 tumors (invad- 
ing local structures) have been subclassified as T4a and $\mathrm{T} 4 \mathrm{~b}$. T4a tumors are considered resectable; $\mathrm{T} 4 \mathrm{~b}$ tumors are considered unresectable, invading other adjacent structures, such as the aorta, vertebral body, and trachea. Esophageal tumors include gastroesophageal tumors whose epicenter is at the GEJ, even if they extend to the proximal $5 \mathrm{~cm}$ of the cardia.

In contrast to the previous system, the recent modifications allow for resection of some T4 tumors, specifically $\mathrm{T} 4 \mathrm{a}$, those invading adjacent structures such as pleura, pericardium, or diaphragm. Although the actual surgical practice has not changedsurgeons have long distinguished between tumors invading resectable versus unresectable structuresthe current staging system provides consistency, both in assessing prognosis and assigning therapy to patients with T4 tumors.

The significance of the inclusion of large GEJ tumors, extending up to $5 \mathrm{~cm}$ within the cardia, is unknown. The use of induction therapy and the selection of specific chemotherapy agents may be influenced by this new designation. Additionally, surgeons must take into account the inferior extent into the cardia. Most Siewert type 2 tumors ${ }^{4}$ are resectable with standard approaches to esophagogastrectomy, ${ }^{1}$ but some tumors with significant involvement of the esophagus may also require complete or near complete gastrectomy in addition to esophageal resection, with use of an alternative conduit. Although the staging system itself did not create this change, the fact that the new esophageal classification now includes these tumors must be recognized.

Histologic classifications of tumor grade and location have been included in the new staging system, and are incorporated into stage group designation because of their influence on prognosis. Increasing histologic grade is associated with incrementally decreasing survival for early-stage cancers. For adenocarcinoma, the distinction of G1 and G2 (well and moderately differentiated) from G3 (poorly differentiated) is important for stage I and IIA cancers. Tumor location (upper and middle thoracic vs. lower thoracic) is important for grouping $\mathrm{T} 2-3, \mathrm{NO}, \mathrm{MO}$ squamous cell cancers.

Most patients with HGD may be treated with radiofrequency ablation. ${ }^{5}$ T1a tumors, defined as tumors involving the mucosa but not invading the submucosa, may be considered for endoscopic mucosal resection (EMR) or esophagectomy in experienced centers. ${ }^{6}$ Tumors in the submucosa (T1b) or deeper are treated with esophagectomy. The treatment of patients with T2,NO disease is controversial, with some centers preferring primary surgical therapy with or without adjunct therapy, whereas others prefer induction therapy followed by surgery. Most patients with T3 and T4a tumors should undergo induction therapy, followed by restaging and possible surgical resection. ${ }^{7}$ The location and histologic grade of tumors do not independently dictate resectability but may be taken into account with other factors in determining the role of surgery in a particular patient, such as age, performance status, nodal involvement, and response to induction therapy.

Unresectable esophageal or GEJ cancer should include cervical or cervicothoracic esophageal carcinomas (within $5 \mathrm{~cm}$ of the cricopharyngeus) and $\mathrm{T} 4 \mathrm{~b}$ tumors with involvement of the heart, great vessels, trachea, or adjacent organs, including liver, pancreas, lung, and spleen. These patients should be treated primarily with definitive chemoradiation.

\section{Nodal (N) Status}

A regional lymph node has been redefined to include any paraesophageal node extending from cervical nodes to celiac nodes. In reclassifying the $\mathrm{N}$ substatus according to the number of histologically positive nodes, $\mathrm{N} 0$ represents none, $\mathrm{N} 1$ represents 1 to 2 nodes, N2 represents 3 to 6 positive nodes, and $\mathrm{N} 3$ represents 7 or more positive nodes, consistent with the $\mathrm{N}$ designations for gastric malignancies.

These changes are based on the databases included in the creation of the new staging system, analyzing patients who underwent surgery without induction therapy. Although the changes in the $\mathrm{N}$ substatus designation will improve prognostic stratification for patients treated with primary surgery, the accuracy of the prognostic assessment in patients treated with induction followed by surgery is currently unknown. More importantly, the modifications regarding $\mathrm{N}$ status, although providing consistency with the gastric staging system, do not give guidance regarding the assignment of therapy: which patients should be considered to have a surgically curable tumor based on nodal status?

Although various surgical approaches may be used, adequate lymph node dissection should accompany the resection, either a 2- or 3-field lymph node dissection. ${ }^{8}$ In patients undergoing 
Strong et al

esophagectomy without induction chemoradiation, at least 15 lymph nodes should be removed to achieve adequate nodal staging. The optimum number of nodes after preoperative chemoradiation is unknown, although similar lymph node resection is recommended. ${ }^{9}$

\section{Metastasis (M) Status}

The subclassifications M1a and M1b have been eliminated, as has MX, and distant metastases are now designated M0 (no distant metastasis) and M1 (any distant metastasis). Although the previous staging system allowed for surgical resection in selected patients with "M1a" disease, the new version is more consistent with the use of the $M$ status. Decision-making regarding patients with celiac node involvement, however, is only shifted to the $\mathrm{N}$ substatus. It is possible that the quantitative aspect of the 7 th edition nodal staging will improve selection for surgical therapy, although the nodal staging itself is based on postoperative pathologic staging (as opposed to information available preoperatively).

\section{Esophageal Summary}

Patients with potentially resectable esophageal cancer should undergo multidisciplinary evaluation. Informed use of the new staging system and treatment guidelines will improve outcomes for patients with esophageal carcinoma. The 7th edition of the AJCC Cancer Staging Manual improves the T designations for T1 and T4 tumors, improves the prognostic stratification of the $\mathrm{N}$ status by incorporating nodal involvement quantitatively, and introduces the parameters of tumor location and grade into the staging system.

Despite the improvements made in the most recent classification of esophageal cancer, several controversial issues still impact treatment guidelines. The new classification remains limited by its source databases, and the ability of the staging system to translate into pretreatment clinical staging or posttreatment pathologic staging for patients who undergo multimodality therapy is currently unknown. Furthermore, because the retrospective analysis of these databases used overall survival, the cancer-specific prognostic stratification of the new staging groups is uncertain.

\section{Gastric Cancer}

The staging classification for gastric cancer also has been limited by several factors, including tumor location and known prognostic variations, heterogeneity of diffuse and intestinal type tumors, inconsistent use of multimodality therapy in operable patients, and differences in outcome among patients in Eastern and Western countries. ${ }^{10}$ The 7 th edition of the AJCC Cancer Staging Manual ${ }^{2,3}$ attempts to harmonize some of the inconsistencies of previous versions. The modifications and areas of controversy of the staging system and the treatment guidelines are described.

\section{Tumor (T) Status}

The classification omits the previous designation HGD and now refers to this as Tis or carcinoma in situ for any intraepithelial tumor without invasion of the lamina propria. These patients should be considered for EMR by experienced centers. For T1 tumors, because a significant chance of lymphatic spread still exists, the T1 category has furthermore been subdivided into T1a (invasion of the lamina propria or muscularis mucosae) and $\mathrm{T} 1 \mathrm{~b}$ (invasion into the submucosa).

Gastric tumors now do not include gastroesophageal tumors whose epicenter is at the GEJ, even if they extend to the proximal $5 \mathrm{~cm}$ of the cardia. This is relevant because although the staging classification has changed, the operative approaches have not. Siewert type 2 tumors $^{4}$ are resectable with standard approaches to esophagogastrectomy, although often tumors with involvement of the esophagus may also require complete or near complete gastrectomy, in addition to esophageal resection.

The new system now uses the TNM system for esophageal carcinoma for tumors arising at the GEJ or in the stomach $5 \mathrm{~cm}$ or less from the GEJ. $\mathrm{T}$ categories now are harmonized with those of the esophagus, with T2 defined as invasion into the muscularis propria, T3 defined as a tumor that invades the subserosal connective tissue, and T4 as defined below.

T4 tumors (invading local structures) have been subclassified as T4a and T4b. T4a tumors invade the serosa (visceral peritoneum) but are considered resectable, whereas $\mathrm{T} 4 \mathrm{~b}$ tumors invade other adjacent structures, such as the spleen, transverse colon, liver, 
diaphragm, pancreas, abdominal wall adrenal gland, kidney, small intestine, and retroperitoneum. In contrast to previous staging systems, the recent modification allows for resection of some T4 tumors, specifically T4a, those invading visceral peritoneum. This enables improved harmonization of surgeons' practice of resecting tumors that invade the serosa but less often offering resection for invasion of adjacent organs. The current staging system also provides consistency for gastric cancer, both in assessing prognosis and assigning therapy to patients with T4 tumors.

Most patients with T1a tumors, defined as tumors involving the mucosa but not invading the submucosa, may be considered for EMR or esophagectomy in experienced centers. ${ }^{6}$ Tumors in the submucosa (T1b) or deeper are treated with gastrectomy with modified D2 lymphadenectomy. The treatment of patients with T2,NO disease varies, with some centers preferring primary surgical therapy with or without adjuvant therapy, and others preferring neoadjuvant therapy followed by surgery. Medically fit patients with T3 and T4a tumors should undergo neoadjuvant therapy after completion of staging by laparoscopy and peritoneal lavage, followed by restaging and possible surgical resection. ${ }^{11}$ The location and histologic grade of tumors do not independently dictate resectability, but may be taken into account with other factors in determining the role of surgery in a particular patient, such as age, performance status, nodal involvement, and response to neoadjuvant therapy.

\section{Nodal (N) Status}

$\mathrm{N}$ status has been reclassified to harmonize with the esophageal system. In reclassifying the $\mathrm{N}$ substatus according to the number of histologically positive nodes, N0 represents none, N1 represents 1 to 2 positive lymph nodes, N2 represents 3 to 6 positive lymph nodes, and $\mathrm{N} 3$ represents 7 or more positive nodes, consistent with the $\mathrm{N}$ designations for esophageal malignancies.

These changes are also based on the extensive Western and Eastern databases that helped better define the new staging system, analyzing patients who underwent surgery without induction therapy. Although the changes in the $\mathrm{N}$ substatus designation will improve prognostic stratification for patients treated with primary surgery, and likely encourage more significant adjuvant treatment for patients, the accuracy of these assessments for patients who have undergone surgery after neoadjuvant treatment is still unknown, and this will require careful analysis of prospectively collected data to further refine indications.

Generally, multistation bulky lymphadenopathy is considered a relative contraindication to surgical intervention, although response to neoadjuvant treatment and a patient's functional status are important factors in recommending possible surgery. However, data suggest that to optimally stage a patient with gastric and esophageal cancer, a minimum of 15 lymph nodes should be removed to achieve adequate nodal staging [Brennan]. How preoperative treatment affects lymph node retrieval and prognosis remains unknown.

\section{Metastasis (M) Status}

A major change in gastric cancer staging in the 7th edition is the inclusion of positive peritoneal cytology as M1 disease. This is an important addition that adds greatly to the management of patients who may otherwise undergo futile operations. Data from Eastern and Western countries have shown that patients with positive peritoneal cytology have outcomes identical to those of patients with visible M1 disease or distant metastases. ${ }^{11}$ Therefore, patients clinically staged as locally advanced (using endoscopic ultrasound) are recommended to undergo diagnostic laparoscopy before initiation of neoadjuvant treatment to complete staging and rule out metastatic disease. Using this method, up to $16 \%$ of patients with otherwise occult metastatic disease can be identified and appropriately treated with avoidance of a potentially morbid gastrectomy. ${ }^{12}$

\section{Gastric Summary}

Patients with potentially resectable gastric cancer should undergo multidisciplinary evaluation. The new staging system and treatment guidelines will improve outcomes for patients with esophageal carcinoma. The 7th edition of the AJCC Cancer Staging Manual improves the $\mathrm{T}$ designations for $\mathrm{T} 1$ and $\mathrm{T} 4$ tumors, improves the prognostic stratification of the $\mathrm{N}$ status by incorporating nodal involvement quantitatively, and has added clearer definitions for M1 disease to optimize treatment options.

Despite the improvements made in the most recent classification, several controversial issues still impact treatment guidelines. The most significant one relates to GEJ tumors. 
GEJ Issues: Another important change in the recently adopted 7th AJCC/Union Internationale Contre le Cancer (UICC) staging system for esophageal and gastric cancer is a clearer definition of GEJ tumors. This presents a frequent controversy for surgical and medical oncologists, and similar patients with involvement just above or below the GEJ may be managed either with esophageal- or gastric-based chemotherapy regimens and radiation fields. In the new staging system, GEJ tumors are more precisely defined as a cancer whose epicenter is in the distal thoracic esophagus, GEJ, or within the proximal $5 \mathrm{~cm}$ of the stomach (cardia) that extends into the GEJ or distal thoracic esophagus.

This clearer definition of the GEJ should allow better data collection in the future, with potential to guide treatment for individual patients, but what does the new staging system mean for patients seeking treatment today? Definitive trials compare outcomes with each approach versus surgery alone, showing a benefit justifying their use; however, these distinct approaches have not been directly compared with one another nor have enough GEJ tumors been treated to power a direct comparison. Only accumulation of data on outcome and patterns of failure for each stage, particularly in patients undergoing neo/adjuvant treatment, will allow better classification in the future to guide treatment, because the 7th AJCC/UICC staging system was based on outcome for surgery alone as the therapy.

In addition, the diagnosis adenocarcinoma and squamous cell is included in the esophageal staging. These histologies were originally grouped together because the prognosis seemed similar, but with the introduction of novel chemotherapy agents and targeted agents, treatment strategies by stage will likely diverge. For example, when developing treatment strategies, the curative potential of 5-FU/ cisplatin/radiation without surgery as definitive therapy was confirmed in RTOG 85-01 for patients with squamous cell carcinoma (often treated nonoperatively at other body sites), but it is more controversial in patients with adenocarcinoma. ${ }^{14}$ Indeed, surgery should be utilized whenever reasonable for adenocarcinoma, because outcome data for definitive chemoradiation are still limited (adenocarcinoma is rarely treated nonoperatively at other body sites). These questions are of importance because the incidence of GEJ adenocarcinoma is rapidly increasing in Western countries.
The new staging system has brought a change in $T$ staging, T1 and T4, as described earlier. Patients with T1 disease in this subset will also likely be managed with surgical options alone, because the prognosis is excellent unless other adverse factors are present. T2 without nodal involvement is more controversial and may be treated with adjuvant or combined modality therapy. Generally, patients with higher $\mathrm{T}$ stages are treated with adjuvant or neoadjuvant therapy. T4 is now divided into $\mathrm{T} 4 \mathrm{a}$, which would be resectable under many circumstances, and $T 4 b$, which are generally unresectable.

The change in the $\mathrm{N}$ staging system is currently of uncertain importance in developing treatment pathways, because even patients with pathologic N1 disease under the new system have poor enough outcome to justify additional treatment. In a series of 2920 surgically treated patients, ${ }^{14}$ - and 10 year survivals were $34 \%$ and $24 \%$, respectively, for N1 (1-2 nodes) disease, sufficient to motivate use of toxic combined modality therapies to improve outcomes. Previously, any patient with nodal disease was staged as N1 and was often, depending on their clinical circumstances, considered a candidate for combined modality therapy. Although continuing this approach for any patient with $\mathrm{N}+$ disease seems reasonable, data will be needed to confirm a benefit, especially because celiac axis nodes are now considered regional nodes rather than M1a disease.

Surgery alone may be appropriate for many patients with early disease, poor performance status, or concurrent illness increasing the risks of complications. In many randomized trials, patients with GEJ tumors represented only a minority and were not reportable as an appropriately powered subgroup, whereas recently the incidence of these tumors has been increasing. For preoperatively treated patients, the approaches for determining stage were generally less accurate than those that may be applied today, such as endoscopy with ultrasound, staging laparoscopy, and PET scanning.

Summary of GEJ Tumors: The new staging system may facilitate better study of GEJ tumors and allow for easier comparison of trial results to refine later study. Having a coherent definition of GEJ tumors is especially important, because they have been treated as esophageal or gastric tumors, depending on the approach of individual treatment teams. Although 
GEJ tumors meeting the specified criteria are now considered esophageal tumors, systemic and radiation therapy must be optimized for this site.

Another important element in the new 7 th AJCC/UICC staging system is the recognition of adenocarcinoma and squamous cell carcinoma as separate entities that may ultimately benefit from differing treatment approaches. Definitive chemoradiation is already known to be potentially curative in squamous cell tumors, but data about adenocarcinoma are limited. More information may be provided by RTOG 0436, an ongoing phase III trial evaluating the addition of cetuximab to paclitaxel, cisplatin, and radiation for patients with esophageal cancer who are treated without surgery. It is highly likely that these histologies will respond differently to some systemic chemotherapies and targeted agents.

Because the new system was based on large data sets of patients treated with surgery alone, what the changes will mean is uncertain. Until more data are available, it is appropriate to continue similar treatment patterns based on studies guiding treatment during earlier versions of the staging system. For patients with locally advanced GEJ tumors, several options are appropriate. Certainly surgical resection followed by chemoradiation can be efficacious even though specific data for the GEJ subgroup are not available. Pre- and postoperative chemotherapy remains another option, as is neoadjuvant chemoradiation followed by later adjuvant chemotherapy when tolerated.

\section{Overall Conclusions}

The 7th edition of the AJCC Cancer Staging Manual has improved harmonization of gastric and distal esophageal/GEJ-type adenocarcinomas, although issues persist, particularly regarding the optimal neoadjuvant treatment for managing GEJ carcinomas. Many outstanding controversies reflect the refined understanding of the heterogeneity of these tumors. The new staging system has achieved several goals, including recognition of the complex biologic diversity of gastric and distal esophageal/GEJ tumors and the recognition of the biologic diversity of these various tumor types. This understanding will help future versions of the AJCC Cancer Staging Manual and NCCN Clinical Practice Guidelines in Oncology to better characterize these subtypes of gastric and esophageal/GEJ tumors, and will facilitate data collection and analysis to better address these discrepancies.

The addition of cancer-specific survival in future versions will make the next proposed staging more accurate. In addition, the inconsistency of surgical approaches, especially with respect to nodal resection, remains a barrier to staging patients after resection, despite whether induction therapy is involved. The collaboration between Eastern and Western high-volume centers in gastric cancer will encourage better evaluations of these subtypes, which will further understanding of the pathogenesis of the various forms of gastric cancer and the optimal treatment. Recognition of the importance of thorough surgical resection and pathologic analysis will improve the staging, and perhaps outcome, of patients with resectable esophageal and gastric cancer.

\section{References}

1. Ajani JA, Barthel JS, Bekaii-Saab T, et al. Esophageal cancer. J Natl Compr Cancer Netw 2008;6:818-849.

2. Edge SB, Byrd DR, Compton CC, et al, eds. AJCC Cancer Staging Manual, 7th ed. New York, NY: Springer-Verlag; 2009:103-115.

3. Rice TW, Blackstone EH, Rusch VW. 7th Edition of the AJCC Cancer Staging Manual: esophagus and esophagogastric junction. Ann Surg Oncol 2010;17:1721-1724.

4. Siewert JR, Stein HJ. Carcinoma of the cardia: carcinoma of the gastroesophageal junction-classification, pathology and extent of resection. Dis Esophagus 1996;9:173-182.

5. Shaheen NJ, Sharma P, Overholt BF, et al. Radiofrequency ablation in Barrett's esophagus with dysplasia. N Engl J Med 2009;360:22772289.

6. Fujita H, Sueyoshi S, Yamana H, et al. Optimum treatment strategy for superficial esophageal cancer: endoscopic mucosal resection versus radical esophagectomy. World J Surg 2001;25:424-431.

7. Urschel J, Vasan H. A meta-analysis of randomized controlled trials that compared neoadjuvant chemoradiation and surgery to surgery alone for resectable esophageal cancer. Am J Surg 2003;185:538543.

8. Hofstetter WL. Lymph node dissection in esophageal cancer. Current therapies in thoracic and cardiovascular surgery. In: Yang SC, Cameron DE, eds. Philadelphia, PA: Mosby, Inc.; 2004:360_ 363.

9. Rizk N, Venkatraman E, Park B, et al. The prognostic importance of the number of involved lymph nodes in esophageal cancer: implications for revisions of the American Joint Committee on Cancer staging system. J Thorac Cardiovasc Surg 2006;132:13741381.

10. Strong VE, Song KY, Park CH, et al. Comparison of gastric cancer survival following RO resection in the United States and Korea using an internationally validated nomogram. Ann Surg 
Strong et al

\section{0;251:640-646.}

11. Mezhir JJ, Shah MA, Jacks L, et al. Positive peritoneal cytology in patients with gastric cancer: natural history and outcome of 291 patients, Ann Surg Oncol 2010;17:3173-3180.

12. Karpeh MS, Leon L, Klimstra D, Brennan MF. Lymph node staging in gastric cancer: is location more important than number? An analysis of 1038 patients. Ann Surg 2000;232:362-371.
13. Sarela AI, Miner TJ, Karpeh, MS, et al. Clinical outcomes with laparoscopic stage M1 unresected gastric adenocarcinoma. Ann Surg Onc 2006;243:189-195.

14. Cooper JS, Guo MD, Herskovic A, et al. Chemoradiotherapy of locally advanced esophageal cancer: long-term follow-up of a prospective randomized trial (RTOG 85-01). Radiation Therapy Oncology Group. JAMA 1999;281:1623-1627. 